\title{
Simple Models of Plant Learning and Memory
}

\author{
Indrani Bose* and Rajesh Karmakar\# \\ 30th October 2018 \\ Department of Physics \\ Bose Institute \\ 93/1, A.P.C. Road \\ Kolkata-700 009, India
}

\begin{abstract}
Plants are capable of intelligent responses to complex environmental signals. Learning and memory play fundamental roles in such responses. Two simple models of plant memory are proposed based on the calcium-signalling system. The memory states correspond to steady state distributions of calcium ions.

P.A.C.S. Nos.: 05.10.-a, 05.45.-a, 87.16.-b, 87.17.-d
\end{abstract}

*email-indrani@bic.boseinst.ernet.in

\# email-rkarmakar2001@yahoo.com 


\section{Introduction}

Plants have long been regarded as passive organisms since they do not possess brains, cannot speak and lack in motility. Plants, on the other hand, constitute $99 \%$ of the biomass of the earth demonstrating their adaptive ability to survive in widely different environments. In recent years, there is a growing belief that plants are capable of intelligent responses to environmental stimuli [1, 2]. Intelligence can be defined as adaptively variable behaviour in the lifetime of an organism. It is in this sense that one can speak of plant intelligence. Plant behaviour is remarkably complex in that plants exhibit considerable flexibility in their responses, have foresight and can anticipate future problems [3]. To give a specific example, the parasitic plant dodder can assess the exploitability of a host soon after their initial contact. The dodder coils about the host with a specific number of turns and sends in a number of tentacles depending on its assessment of possible future yield from the host. Aspects of intelligent behaviour include the ability to compute, to learn and to retain memory. Similarities between the neuronal network of brains and calcium signalling systems in plants have been pointed out [2, 4. There are many examples of crosstalk, i.e., connections between the biochemical signalling pathways in plants. A Boolean representation of the networks of signalling pathways is possible in terms of well-known logical gates like AND, OR, NAND, NOR, XOR, and XNOR [4]. The Boolean description makes it possible to draw analogies between plant signalling networks and computing devices. Recently, an electrical network model of plant intelligence has been proposed which can perform logical operations [5].

There are close parallels between a neural network and a calcium signalling system. The former is a network of neurons or nerve cells. The network has

a complex structure as each neuron is connected to a large number of other neurons. The input to a neuron is in the form of electrical pulses from the neurons to which it is connected. If the resultant sum exceeds a threshold value, the neuron fires ("on" state) and sends out electrical pulses to the other connected neurons through axons and synaptic junctions [6, 7]. The inactive state of the neuron is known as the "off" state. In a calcium signalling system, one has a network of ion channels which may be located on the outer plasma membrane of the cell or on the membranes of intracellular vesicles and organelles. Like the neurons, an ion channel can be in two states, closed or open. The normal concentration of free $\mathrm{Ca}^{2+}$ in 
the cytoplasm is much lower than that in the extra-cellular fluid and in the intra-cellular vesicles and organelles. When a cell receives an input signal, a series of biochemical events constituting the signalling pathway are initiated leading to a rapid rise in the concentration of the sugar phosphate inosital 1,4,5-triphosphate $\mathrm{IP}_{3}$ in the cytoplasm. $\mathrm{A} \mathrm{Ca}^{2+}$ ion channel opens when both $\mathrm{IP}_{3}$ and $\mathrm{Ca}^{2+}$ bind at appropriate sites of the channel. Through the open channel, $\mathrm{Ca}^{2+}$ ions move from the interior to the exterior of the membrane. The released calcium ions diffuse to neighbouring channels and open them up giving rise to further calcium release. The coordinated release of calcium ions gives rise to a calcium wave in the network. The flow of calcium wave is analogous to the transmission of electrical pulses in a neural network. Long before the calcium signalling pathway was identified, J. C. Bose through his pioneering experiments showed that plant cells are excitable and can transmit millivolt order electrical signals at the speed of $10-40 \mathrm{~mm} / \mathrm{sec}$. 8]. Even after Bose's experimental observations, the prevalent belief amongst plant physiologists was that cell signalling involves solely chemical diffusion. With the elucidation of the calcium signalling pathway, it is now well established that the release and subsequent diffusion of $\mathrm{Ca}^{2+}$ ions gives rise to propagating electrical pulses in the cellular network thus vindicating Bose's earlier prediction.

Synaptic transmission is not the exclusive mechanism for neurotransmission in a neural network. There is increasing evidence that non-synaptic diffusion neurotransmission plays a significant role in some brain functions [9]. Reaction-diffusion (RD) processes involving antagonistic neurotransmitters can give rise to spatio-temporal organization in the neural network. RD systems using only local interactions have been shown to give rise to wide-ranging phenomena including travelling waves, oscillations and formation of stationary patterns [10, 11]. In this paper, we discuss two simple RD-type models of networks of calcium ion channels and show that spatiotemporal organization takes place in the steady state. The possible role of such organization in plant learning and memory is further pointed out. Since learning and memory are attributes of intelligence, our model studies can be considered as tentative attempts to obtain insight on the complexities of plant intelligence. 


\section{Reaction - diffusion models of calcium signalling networks}

The first model is a minimal model incorporating some of the essential features of calcium cell signalling. We consider a network of ion channels in one dimension (1D). Each channel can exist in two possible states: inactive and active. In the inactive state a channel is closed and in the active state a channel is open. An open channel allows for the release of $\mathrm{Ca}^{2+}$ ions from the internal store of the channel. The model dynamics are described in terms of two concentration variables, $u_{1}$ and $u_{2}$, denoting the concentrations of active channels and calcium ions respectively. Detailed processes, like $\mathrm{IP}_{3}$ and calcium binding, leading to the opening of the channel are ignored. An active channel can become inactivated on the binding of calcium ion to an inactivating site. $\mathrm{Ca}^{2+}$ can diffuse through the network of channels and on binding at appropriate sites can activate/inactivate the channels. The concentration of active channels at a particular location can change due to changes in the concentrations of active channels in neighbouring regions. This effect is represented through a "diffusive" coupling, the diffusion in this case refers to the propagation of channel activity.

The dynamics of the model are described by the following differential equations:

$$
\begin{aligned}
& \partial_{t} u_{1}=D_{1} \partial_{x}^{2} u_{1}+a_{1} u_{1}-b_{1} u_{2} \\
& \partial_{t} u_{2}=D_{2} \partial_{x}^{2} u_{2}+b_{2} u_{1}-a_{2} u_{2}
\end{aligned}
$$

In Eq.(1), $D_{1}$ represents the effective "diffusion constant" for the propagation of channel activity. The rate of change of the concentration of active channels is assumed to be linearly proportional to the concentration of active channels. The release of $\mathrm{Ca}^{2+}$ ions through open channels can activate further opening up of calcium channels. This effect is indirectly incorporated through the second term in Eq. (1). The third term in Eq.(1) represents explicitly the role of $\mathrm{Ca}^{2+}$ ions in inactivating ion channels and thereby decreasing $\partial_{t} u_{1}$. The first term in Eq.(2) describes the diffusion of $\mathrm{Ca}^{2+}$ ions, $D_{2}$ being the diffusion constant. The second term shows that $\partial_{t} u_{2}$ increases if the concentration $u_{1}$ of active channels increases. The third term is a decay term and arises out of the pumping back of $\mathrm{Ca}^{2+}$ ions into the internal stores. 
In a remarkable paper in 1952, Turing showed that a RD system involving a slowly-diffusing activator and a fast-diffusing inhibitor can give rise to a pattern forming instability [12, 13] in which stationary patterns are formed in the non-equilibrium steady state. RD systems with dynamics of the type described in Eqs.(1) and (2) can exhibit different types of instability including the Turing-type [12]. We provide the details below.

In Eqs.(1) and (2), $u_{1}$ and $u_{2}$ represent the concentrations of the activator (active channels) and inhibitor $\left(\mathrm{Ca}^{2+}\right)$ respectively. The growth of $u_{1}$ stimulates the growths of $u_{1}$ and $u_{2}$. On the other hand, as the name implies, a rise in the concentration $u_{2}$ of the inhibitor inhibits the growths of $u_{1}$ and $u_{2}$. The inhibitor diffuses further than the activator, i.e., $D_{2}>D_{1}$. In the absence of diffusion, the steady state of Eqs.(1) and (2) is given by

$$
\left(u_{1 s}, u_{2 s}\right)=(0,0)
$$

The stability conditions from linear stability analysis are:

$$
\begin{gathered}
a_{1}-a_{2}<0 \\
\operatorname{det} A=b_{1} b_{2}-a_{1} a_{2}>0
\end{gathered}
$$

One would now like to determine whether the homogeneous steady state becomes unstable on inclusion of the diffusion terms in Eqs.(1) and (2). Define a two-component columns vector $u$ with elements $u_{1}$ and $u_{2}$. We look for solutions of the form $u \sim e^{w t} e^{i k x}$. Linearising Eqs.(1) and (2) about the steady state in (3), one gets the following characteristic equation for $w$ :

$$
w^{2}-T w+\Delta=0
$$

where

$$
T=a_{1}-a_{2}-\left(D_{1}+D_{2}\right) k^{2}
$$

and

$$
\Delta=b_{1} b_{2}-a_{1} a_{2}-k^{2}\left(a_{1} D_{2}-a_{2} D_{1}\right)+D_{1} D_{2} k^{4}
$$

The solutions of the characteristic equation are

$$
w_{ \pm}=\frac{1}{2}\left[T \pm \sqrt{T^{2}-4 \Delta}\right]
$$


The steady state $\left(u_{1 s}, u_{2 s}\right)$ is linearly stable if $\operatorname{Re}\left(w_{ \pm}\right)$is $<0$. We have already imposed the conditions, Eqs.(4) and (5), that the steady state is stable in the absence of diffusion, i.e., Re $w_{ \pm}\left(k^{2}=0\right)$ is $<0$. If the steady state is to be unstable to spatial disturbances, Re $w_{+}(k)>0$ for some $k \neq 0$. This is true if either $T$ is $>0$ or if $\Delta\left(k^{2}\right)<0$ for some $k \neq 0$. Due to the condition in Eq.(4), $T$ is always less than zero. The condition in Eq.(5) demands that the only possibility for $\Delta\left(k^{2}\right)$ to be negative is if $\left(a_{1} D_{2}-a_{2} D_{1}\right)>0$. Since $a_{1}<a_{2}$ (Eq.(4)), one gets the additional condition

$$
D_{2}>D_{1}
$$

That is, the inhibitor diffuses faster than the activator. For $\Delta\left(k^{2}\right)$ to be negative for some non-zero $k$, the minimum value of $\Delta, \Delta_{\text {min }}$, must be negative. Differentiating Eq.(8) w.r.t. $k^{2}$, one gets

$$
\Delta_{\text {min }}\left(k^{2}\right)=b_{1} b_{2}-a_{1} a_{2}-\frac{\left(a_{1} D_{2}-a_{2} D_{1}\right)^{2}}{4 D_{1} D_{2}}
$$

with

$$
k^{2}=k_{m}^{2}=\frac{a_{1} D_{2}-a_{2} D_{1}}{2 D_{1} D_{2}}
$$

Thus the condition that $\Delta\left(k^{2}\right)<0$ for some $k^{2} \neq 0$ is

$$
\frac{\left(a_{1} D_{2}-a_{2} D_{1}\right)^{2}}{4 D_{1} D_{2}}>b_{1} b_{2}-a_{1} a_{2}
$$

To summarize, if the conditions (4), (5), (10) and (13) are satisfied, the homogeneous steady state is unstable towards a stationary state with wave number

$$
k_{m}=\left[\frac{1}{2}\left[\frac{a_{1}}{D_{1}}-\frac{a_{2}}{D_{2}}\right]\right]^{\frac{1}{2}}
$$

At the bifurcation point, $\Delta_{\min }=0$. Since $\Delta=w_{+} w_{-}$, this implies that one of the roots of the characteristic equation is zero. The bifurcation can be brought about by changing the parameters of the system. In the steady state, stationary distributions of the activator and inhibitor concentrations are obtained.

In the simple network model of calcium ion channels considered by us, the condition (10) implies that $\mathrm{Ca}^{2+}$ ions diffuse faster compared to the "diffusion" of channel activity. This is plausible in a real calcium signalling 
system as for a channel to be activated by neighbouring channels, the released $\mathrm{Ca}^{2+}$ ions from these channels have to diffuse to the channel in question followed by the binding of $\mathrm{IP}_{3}$ and $\mathrm{Ca}^{2+}$ ions at the appropriate channel sites. The steady state stationary distributions are analogous to the attractors (memory states) in the case of a neural network. Figure 1 shows the steady state distributions of $u_{1}$ and $u_{2}$ in an 1d lattice of 50 sites with periodic boundary conditions. The distributions have been obtained by solving Eqs.(1) and (2) numerically on discretizing the derivative terms in the two equations in the Euler scheme. The values of $\Delta x$ and $\Delta t$ have been chosen to be 1 and 0.01 respectively. The discretization can be treated as a simple approximation to the partial differential Eqs. (1) and (2). The finite difference equation may alternatively be treated as a representation of the $\mathrm{RD}$ system on a lattice. The initial state of the lattice is the $\left(u_{1}, u_{2}\right)=(0,0)$ state perturbed by small random amounts at all the lattice sites.

In a neural network, learning and memory are interlinked. Networks learn through reinforcement of pathways connecting signals to response. One way in which reinforcement can occur is through increasing the strength of existing synaptic connections between neurons. As pointed out by Trewavas 2, learning and memory are also interrelated in the calcium signalling system. On receiving an input signal, the cellular content of the molecules (like $\mathrm{IP}_{3}$ ) participating in the signalling pathway is increased by a large amount. This is an example of cellular learning leading to an accelerated information flux along calcium dependent pathways. Memory of previous signals in a network can be accessed to transform current signals. A calcium-based memory corresponds to an inhomogeneous stationary distribution of $\mathrm{Ca}^{2+}$ ions. The calcium wave generated by a new signal will propagate preferentially in those regions where calcium concentration is high. The final distribution of calcium will be the outcome of the integration of the current signal modified by a stable, long term memory. In our model, the inhomogeneous stationary distribution of calcium which constitutes long term memory is an outcome of the Turing instability. The signal modified by previous memory activates a unique combination of effector proteins which ultimately brings about the desired response to the input . Trewavas has further pointed out that important aspects of the cell memory are possibly associated with the cell wall as its removal affects many of the developmental processes in algae and higher plants. It is well-known that calcium signalling networks can exhibit limit-cycle behaviour in the form of oscillations in the $\mathrm{Ca}^{2+}$ concentration [14]. In the 
model considered by us, the homogeneous steady state (Eq.(3)) undergoes a Hopf bifurcation when $a_{1}>a_{2}$ 12. In this case, $w_{ \pm}$(Eq.(9)) are purely imaginary, i.e., $T=0$ (Eq.(7)). Since the solution for $u$ contains the factor $e^{w t}$, stable oscillations are obtained in the system.

Microorganisms like bacteria and bacteriophage share a common feature with plants, namely, the absence of brains. A recent study [15] has discussed evidence that microorganisms exhibit memory. Memory implies systems the present state of which is not entirely determined by present conditions but depends on past history, i.e., on the path by which the present state is reached. Several examples of history dependence in biological systems are known [15. Most of these systems have two or more stable steady states, the so-called memory states. The choice of a particular state depends on the pathway followed to reach it. Similar examples of memory in a calcium signalling system can be given. Ref.[12 discusses a simple model of calcium-stimulated calcium release the kinetics of which is described by the rate law

$$
\frac{d x}{d t}=\frac{k_{1} x^{2}}{k_{2}+x^{2}}-k_{3} x
$$

where $x$ denotes the concentration of $\mathrm{Ca}^{2+}$ ions. The first term on the r.h.s describes the autocatalytic release of calcium ions (more calcium ions imply more open channels leading to further increases in calcium concentration). The autocatalytic production saturates for high concentrations of $\mathrm{Ca}^{2+}$. The second term represents the pumping back of calcium ions into internal stores. There are three steady states of the system with concentrations given by $x_{1}, x_{2}$ and $x_{3}$ respectively. The first and the third states are stable steady states and the second state is unstable. For all $0<x<x_{2}, x \rightarrow x_{1}$, whereas for all $x>x_{2}, x \rightarrow x_{3}$. Thus the signalling system exhibits memory of past history. A neural network has multiple steady states because transmissions across synaptic junctions can be both excitatory and inhibitory in nature. A calcium signalling network can also have multiple steady states since $\mathrm{Ca}^{2+}$ ions have both activating and inhibiting effects on the opening of ion channels. 


\section{Concluding remarks}

The models of calcium signalling networks studied in this paper are toy models meant to illustrate the origin of memory states in plants in analogy with similar states in neural networks. In reality, the ion channels have a complex three subunit structure and a channel is open only if all the three subunits are simultaneously open [16]. Furthermore, the dynamics are governed by non-linear rate equations. Work on a more realistic model of the calcium signalling network is in progress and the results will be reported elsewhere. Recently some discrete stochastic models of calcium dynamics have been proposed [14, 17. These models address other interesting aspects of calcium signalling networks. In one such model defined on a $1 \mathrm{~d}$ lattice, a set of probabilities for the opening/closing of calcium channels is assumed to depend on the calcium concentration. By increasing the number of channels/site, a transition from a non-propagating region of activity to a propagating one occurs. The transition belongs to the directed percolation class of similar transitions. To sum up, calcium signalling networks present us with a rich array of problems ranging from cellular learning/memory to novel phenomena arising out of activated dynamics.

ACKNOWLEDGEMENT

I. B thanks Bikas K. Chakrabarti, Bharati Ghosh, Dibyendu Sen Gupta and P. C. Sen for useful discussions. R. K. is supported by the Council of Scientific and Industrial Research, India under Sanction No. 9/15 (239)/ 2002 - EMR - 1.

\section{References}

[1] Trewavas, A., Nature 415, 841(2002)

[2] Trewavas, A., Plant Physiology 120, 1 (1999)

[3] Phillips, H., New Scientist, July 27 issue, 41 (2002)

[4] Genoud, T. and Métraux, J-P., Trends in Plant Science 4, 503 (1999)

[5] Chakrabarti, B. K. and Dutta, O., cond-mat/0210538

[6] Hopfield, J. J., Proc. Nat. Acad. Sci. 79, 2554 (1982) 
[7] Hertz, J., Krough, A. and Palmer, R. G., "Introduction to the theory of neural computation" (Addison-Wesley, Reading, MA 1991)

[8] Bose, J. C., "The Nervous Mechanism of Plants" (Longmans, Green and Co., London 1923)

[9] Liang, P., Phys. Rev. Lett. 75, 1863 (1995)

[10] Cross, M. C. and Hohenberg, P. C., Rev. Mod. Phys. 65, 851 (1993)

[11] Walgraef, D., "Spatio-Temporal Pattern Formation" (Springer - Verlag, New York 1997)

[12] Murray, J. D., "Mathematical Biology" (Springer - Verlag, Berlin 1989)

[13] Turing, A. M., Philos. Trans. R. Soc. London B 237, 37 (1952)

[14] Shuai, J-W. and Jung, P. Biophysical Journal 83, 87 (2002)

[15] Casadesús, J. and D’Ari, R., BioEssays 24, 512 (2002)

[16] Shuai, J-W. and Jung, P., Phys. Rev. Lett. 88, 068102 (2002)

[17] Bär, M., Falcke, M., Levine, H. and Tsimring, L. S., Phys. Rev. Lett. 84, 5664 (2000) 